\title{
Science and the Large Hadron Collider: a probe into instrumentation, periodization and classification
}

\author{
Arpita Roy
}

Published online: 5 September 2012

(C) The Author(s) 2012. This article is published with open access at Springerlink.com

\begin{abstract}
On September 19, 2008, the Large Hadron Collider (LHC) at CERN, Switzerland, began the world's highest energy experiments as a probe into the structure of matter and forces of nature. Just nine days after the gala start-up, an explosion occurred in the LHC tunnel that brought the epic collider to a complete standstill. In light of the catastrophic incident that disrupted the operation of the LHC, the paper investigates the relation of temporality to the cycle of work in science, and raises the question: What kind of methodological value should we ascribe to events such as crises or breakdowns? Drawing upon and integrating classical anthropological themes with two and a half years of fieldwork at the LHC particle accelerator complex, the paper explores how the incident in September, which affected the instrument, acquaints us with the distribution of work in the laboratory. The incident discloses that the organization of science is not a homogenous ensemble, but marked by an enormous diversity of tasks and personnel, at the heart of which lies the opposition of theory and practice, or pure and applied. This opposition not only forms the source and sanction of the intricate division of labor found in high-energy physics, but also provides a satisfactory answer to every question involving the interface of experimental science and engineering skill.
\end{abstract}

Keywords Periodization - Liminality - Division of labor - Material culture · Theory and practice $\cdot$ Highenergy physics

In September 2008, the Large Hadron Collider (LHC) at CERN, Switzerland, began the world's highest energy experiments as a probe into the structure of matter and forces of nature. Amidst great fanfare and publicity on Friday, September 10th, at

\footnotetext{
A. Roy $(\bowtie)$

University of California Berkeley, Berkeley, CA, USA

e-mail: arpita@berkeley.edu
} 
10:28 a.m. the first beam of protons was sent into circulation around the full $27 \mathrm{kms}$ of the underground accelerator. The circulation of the first beam of protons in the LHC marked the culmination of a decade-and-a-half of effort by scientists and engineers to build the most ambitious high-energy physics experiment to date. Just nine days after the gala start-up, however, an explosion occurred in the LHC tunnel, which brought the epic collider to a grinding halt. Inquiries were launched to look into the causes of the explosion and the resulting damage. The occasion led to soul searching within the physics community to apprehend if it was a technical fault or a human error, an unforeseen accident or a routine event. While these concerns suggest the familiar recognition that whatever scope science may realize, it is constituted by limitations, a decisive question meets us in a distinctive and original form here: What constitutes the "normal" work life of a scientific laboratory? In other words, are breakdowns and crises integral to the cycle of work or exceptional events? This question provides us not only with a description of the physical profile or the "organization" of scientific industrial work life, but also with an explanation into its inner character. Such an explanation consists in determining the character of instrumentation and its conditions of existence, establishing the significance of temporality in laboratory research, and clarifying the relation between work and norms in modern science and technology.

In Chapter 10 of Capital, "The Working Day," Marx discovers the mechanisms of capitalist exploitation in the rhythm of daily work, particularly, in the length of the working day. The formulation of a working day is the outcome of a relentless struggle: the rights of the capitalist to prolong the working day to its maximum length, and the rights of the proletariat to reduce it to a reasonable or "normal" duration. Marx then reviews the historical data to establish the correlation between the accumulation of capital and the length of the working day. Examining the evidence of the English Factory Acts from 1833 to 1864, he shows how an intrinsically dynamic or variable working day is set to a definite, a normal, working day through the struggle of capital and labor. However, the absolute limits to the working day are set by the (a) physical and (b) social bounds of the labor force. The labor force can only work so many hours in a day and needs physical rest to replenish itself. Besides these physical limitations, the extension of the working day encounters social, moral or intellectual bounds. These intellectual demands, which must be satisfied in order to be alive, are determined by the social conditions of the time (Marx and Engels 1967).

Now if we set aside the overwhelming issue of the conflict between the bourgeoisie and the proletariat, we succeed in extracting from Marx's analysis of a working day, the snapshot of a complex relation between productivity of labor and norms of social life. The recognition of the relation between physical output and social norms is only an element in a great comprehensive analysis which, however, far from being exhausted or obliterated in relation to the total content of his analysis, serves as an indispensable methodological tool especially when we apply it to the technical procedures of science. It allows us to problematize the temporal dimensions of scientific industrial work life. To be sure, temporality in the sense of duration is a basic component and tendency of all scientific industrial work. So considered, temporality remains a prerequisite for every description and analysis 
(Pickering 1984; Traweek 1988). However, to isolate the concept as a measure of social life (of science), we must investigate the external marks of temporality in the morphology and development of tasks undertaken in the laboratory. From this perspective, what matters is (a) the material culture of the laboratory, such as the commissioning and operation of complex instrumentation, (b) to trace it to its intellectual nerve, the procedures and principles that issue from the domain of engineering, which assure us of the integrity, reliability and significance of all instrumentation and (c) the comprehensive culture of high-energy physics, including the institutionalized division of labor among theory, experiment and instrumentation (Galison 1997; Knorr-Cetina 1999).

The paper explores the temporal dimensions of techno-scientific work life in the light of the catastrophic incident that disrupted the operation of the LHC in September 2008. The incident and the resulting suspension of the machine invite us to examine a critical question, and upon whose decision our interpretation of the normative character of science rests: What kind of methodological value should we ascribe to events such as crises or breakdowns? Do they merely have a functional import? Or do they disclose some systematic feature of science? The urge to address these questions will take into account the empirical, with which the paper begins, but also the possibility of an epistemological conception, where at stake is the deeper problem of how temporal events and processes are connected to general principles of knowledge and classification (Bourdieu 1977; Gell 1992; Munn 1992). The roots of these principles and classification can be found in the material culture of the laboratory, the discussion of which the second section takes up. The third section examines, by way of contrast, the responses of physicists and engineers to the September incident and shows how the mishap inscribed the symbolic opposition of theory and practice. The fourth section analyzes the specificity of the September incident-as an instance of "transition"- through anthropological concepts of periodization and liminality. What the transition consists in and how it connects to the division of labor found in high-energy physics is explored in the final part of the paper. The paper concludes that even if it is recognized that the primary purpose of the division of labor is functional coordination, the functional calculus rests upon a structural opposition, the opposition of theory and practice, and so the consideration of function leads us back to form.

The paper is a case-centered inquiry and based on two and a half years of ethnographic fieldwork at the site of the accelerator complex at CERN. CERN, or Conseil Européen pour la Recherche Nucléaire, is the largest high-energy physics experimental facility in the world. The LHC is the flagship project of CERN. ${ }^{1}$ Built at a staggering cost of 3.5 billion Swiss Francs, spread over 15 years, the LHC is the highest energy particle accelerator in the world. At a record energy of 14 trillion electron volts, two counter-rotating proton beams collide head-on with each other every 25 nanoseconds (ns). Particle acceleration and collision is based on the principle of mass-energy conversion $\left(E=m c^{2}\right)$ in which fast-moving particles are collided with

\footnotetext{
1 The term "hadron" refers to composite particles, or particles composed of more elementary matter called "quarks." The best known hadrons are protons and neutrons. The LHC accelerates and collides two counter-rotating beams of protons around $27 \mathrm{~km}$, hence its name, the Large Hadron Collider.
} 


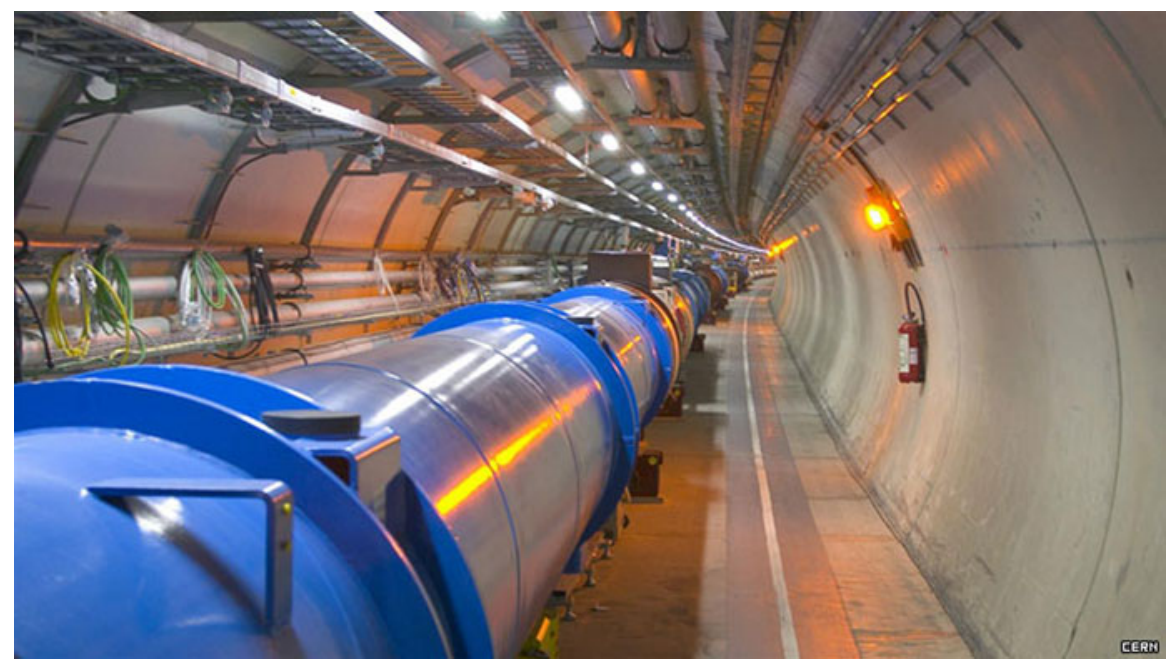

Fig. 1 A cross section of the LHC, showing the dipole magnets, in the underground tunnel

each other so that some of their energy is converted into the creation of exotic new particles. These material collisions and interactions on the LHC are expected to yield extraordinary discoveries into the nature of our universe, including the origin of matter, the explanation of "dark" or invisible matter, uncovering hidden symmetries of the universe, and even finding the possibility of extra dimensions of space.

The collider is housed in a $27-\mathrm{km}$ underground tunnel (Fig. 1). The facility straddles across the French-Swiss border but is officially neither under Swiss nor French jurisdiction. It is privileged with the status of diplomatic immunity. The total personnel employed by CERN is 2,250, out of which approximately 20 are from "Theoretical Physics," some 50 are designated as "Research Physicists," that is, experimentalists who do the job of data analysis, 300 are "Applied Physicists" involved with R\&D (or "research and development"), construction, installation and commissioning pertaining to specific experiments, and over 1,000 engineers, physicists, technicians and mechanics associated with the development and operation of the accelerator itself. The bulk of the workforce, however, does not come directly from CERN but from worldwide universities and research centers that are participating on the various experiments at CERN. Some 8,000 scientists and engineers, representing 580 universities and 85 nationalities, flock here for their research. These features altogether make CERN a rather unique organization. Although an academic establishment-with most people who pass through it holding doctorates-it has the underpinnings of an international corporation with markers of worldly power, success and prestige. Given at once to relatively esoteric concerns of knowledge, truth and endeavor, it is equally savvy with media blitz or "CD" plated cars. ${ }^{2}$

\footnotetext{
2 Vehicles bearing license plates with the letters "CD" that stands for the French term Corps Diplomatique, enjoy a certain level of diplomatic immunity. Generally belonging to vehicles attached to foreign missions, $\mathrm{CD}$ car plates are also given to privileged personnel belonging to international organizations such as senior physicists from CERN as a measure of their importance and rank.
} 
Fig. 2 A badly damaged interconnect splice

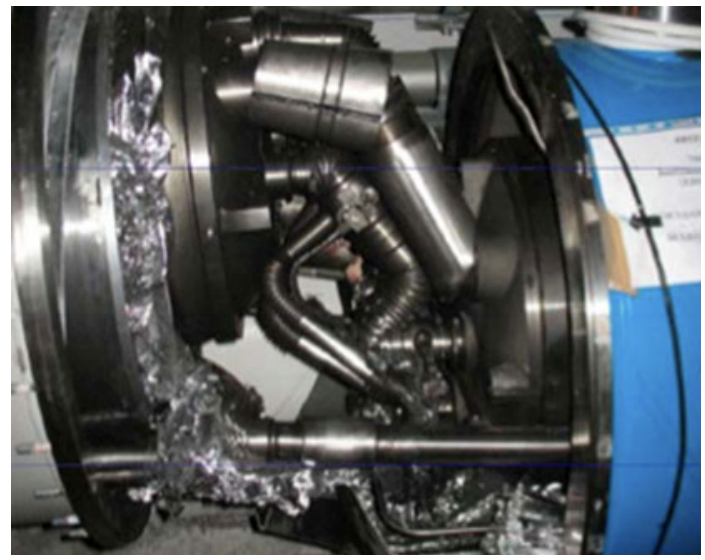

Since August 2007 (until December 2009), I had been conducting anthropological research at CERN in standard participant-observation fashion, interacting and interlocuting with over 100 physicists and engineers. When the mishap occurred on September 19, 2008, I had spent over a year in the physics community and was privy to some of the debates and discussions that took place at the time. Curiously enough, the word "accident" was never used in CERN's press releases or in the media reports to describe the electrical fault and the resulting explosion, presumably because there were no casualties, or because the word accident is more alarming than the term incident. I will keep to the term "incident" consistently throughout to isolate an interesting feature of diachrony of laboratory life, which will disclose the classification of science in its most elementary constitution.

\section{The normal and the exceptional}

On the morning of Friday, September 19, 2008, an electrical connection between two magnets failed during a routine circuit test in sector 3-4 of the LHC ring. At the time, a current of 8.7 Kilo Amperes was being pushed through the superconducting cables. One of the "interconnect splices" linking the cables between two magnets all of a sudden developed resistance and disintegrated-producing an electrical arc-which punctured the containers of liquid helium that keep the magnets in their $1.9 \mathrm{~K}$ (or $-271^{\circ} \mathrm{C}$ ) operating temperature (Fig. 2). Two metric tons of helium gas was instantly released in the LHC tunnel and with such a force that a number of magnets broke their anchors to the concrete floor, and were displaced and damaged beyond recognition (Fig. 3). The release of helium gas tripped the emergency stop thus immediately switching off all electrical power and services from sector 3-4 of the accelerator. ${ }^{3}$

\footnotetext{
3 The LHC circular accelerator is not a perfect circle but is split into eight distinct parts-or sectorscomprising of eight arcs and eight straight sections. The sectors are the working units of the LHC: magnet installation, hardware commissioning, powering, etc., all take place sector by sector.
} 
Fig. 3 A broken magnet support

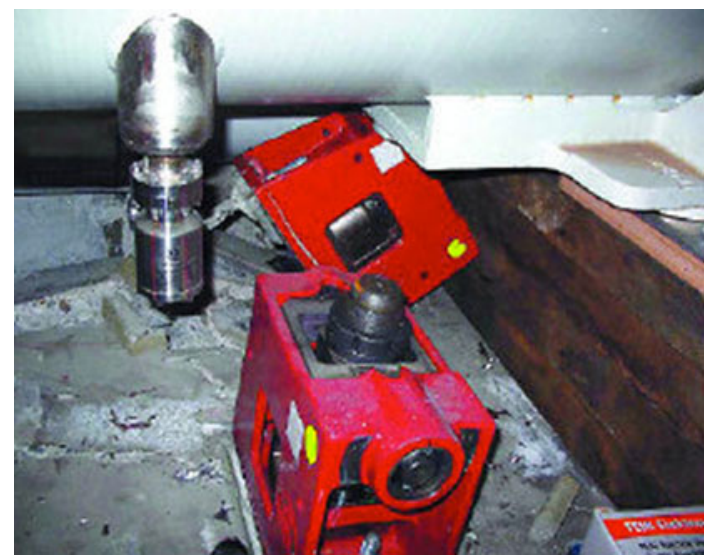

As soon as preliminary news of the incident trickled in, speculations started on the causes and the extent of damage, the amount of time required for the repairs and what that meant for the schedule of the collider's operation. In the first few days surrounding the incident, CERN's management released little information that created some discontentment among sections of scientists. An internal mail from Director General, Robert Aymar, on September 20, 2008, with the subject "incident in LHC sector 3-4" spoke vaguely of "a large helium leak" and "a faulty electrical connection" between two magnets, which "probably melted at high current leading to mechanical failure." A few physicists grumbled that CERN was a research organization—not a diplomatic or a military establishment-and members of the personnel, that is the scientists, had a right to know what had caused the incident since their schedules were going to be affected. They argued how could there be secrecy in an academic environment? Yet others believed that secrecy was necessary so as not to dampen the general morale, or the management must have good reasons for withholding details of the damage, which it would disclose at an opportune moment, or more simply that in an organization so big, everyone cannot know everything right away.

Soon, intense rumors began circulating on the causes and the extent of the damage done to the accelerator. Masco (2002) has spoken insightfully, "secrecy, however, is also wildly productive: it creates not only hierarchies of power and repression, but also unpredictable social effects, including new kinds of desire, fantasy, paranoia, and-above all-gossip" (451). And gossip does not occur in a vacuum, "it is almost always 'plugged in' to social drama" (Turner 1980: 149). Conjectures and speculations on what had led to the calamity, what kind of quality tests had been performed on the "interconnect splices," or why were enough spare parts not available which would hasten the pace of repairs, prefigured most lunchtime conversations as my field notes indicate. It was not uncommon in that period to observe the more aloof theoretical physicists striking conversations with engineers and accelerator physicists in the cafeteria, who had a better idea of what had befallen the accelerator, in attempts to extract information on the status of repairs than was officially available. 
Eventually, the Director General's office released a short report to all CERN personnel on the September incident, received via email on October 16, 2008:

Dear Colleagues, We have today issued an analysis of the 19 September incident at the LHC. Investigations have confirmed that cause of the incident was a faulty electrical connection in a region between two of the accelerator's magnets, which resulted in mechanical damage and release of helium from the magnet cold mass into the tunnel. Proper safety procedures were in force, the safety systems performed as expected, and no one was put at risk. Sufficient spare components are in hand to ensure that the LHC is able to restart in 2009, and measures to prevent a similar incident in the future are being put in place. This incident was unforeseen, but I am now confident that we can make the necessary repairs, ensure that a similar incident cannot happen in the future and move forward to achieving our research objectives. The full report is available here.

https://edms.cern.ch/file/973073/1/Report_on_080919_incident_at_LHC_2_.pdf

Best Regards, Robert Aymar ${ }^{4}$

The Director General's mail and the report disclosed the full extent of the damage to the LHC and immediately led to a general air of depression. The "faulty electrical connection," which had induced the catastrophe, was nothing more than poor soldering! "During repair work in the damaged sector, inspection of the joints revealed systematic voids caused by the welding procedure" Mike Lamont lamented. ${ }^{5}$ It also became clear to everyone that the otherwise minor incident was going to cause a major delay in the reoperation of the machine. The reasons for the delay were the following: 24 of the "dipole" magnets and five "quadrupole" damaged magnets needed to be taken out of the tunnel and sent for repairs. The "interconnect splices" had to be inspected in the remaining sectors. Soot and debris had to be cleaned from the beam pipe. New safety systems and enhanced warning systems had to be installed to prevent similar incidents from happening again. The chief reason for the delay, however, was that the damaged sectors 3-4 of the LHC would have to be warmed up for the inspections and the repairs to take place. Since the LHC is a "superconducting" accelerator that operates at a horribly low temperature of $1.9 \mathrm{~K}$ or $-271{ }^{\circ} \mathrm{C}$, it takes months for the entire $27-\mathrm{km}$ underground tunnel area to regain room temperature, before which maintenance or repair staff cannot enter the tunnel. For a similar fault, not uncommon in a normally conducting accelerator, the repair time would merely be a matter of days. All these factors at the time suggested a minimum of six months downtime for the LHC operation.

\footnotetext{
4 This email was sent by Robert Aymar to all CERN personnel with the subject: Report on 19th Sept 2008 incident at LHC-rapport sur l'incident du 19 Sept 2008 au LHC.

5 The "LHC status report" was presented by Mike Lamont on April 23, 2010. Lamont is the Leader of the Operations Group (Beams Department) who is responsible for the overall technical infrastructure and operation of all CERN present and future accelerators.
} 
The engineering constraints, which decided the timeline of the repairs and the renewed operation, were going to affect the physics situation adversely. The key factor motivating the endless rounds of discussions on spares and repairs was the question mark placed on the LHC physics program and what that meant for the prospects of the rival collider, the Tevatron, at Fermi National Accelerator Laboratory, or Fermilab, Illinois, USA. Any setback in the LHC schedule implied an immediate advantage for the rival collider, the Tevatron. Operating since 1987, the Tevatron was working at peak performance and the September incident suddenly created a palpable possibility that it could overtake the LHC in staking the first claims to a discovery, such as the discovery of the Higgs particle. The BBC ran an interesting article with the title, "Race for God Particle Heats Up." When the news trickled in March 2009 that the Tevatron had excluded the Higgs mass in the window of 160-170 GeV, it created a commotion at CERN. Could the Americans snatch the prize of the Higgs right under the noses of the Europeans? The Tevatron had a head start, their technology was stable, and with three inverse femtobarns of collision data-the scientific unit that scientists use to count the number of collisions-they could use it to blow the LHC out of the water. "Coming immediately after the very successful start of LHC operation on 10 September, this is undoubtedly a psychological blow," bemoaned CERN's Director General, Robert Aymar.

The trans-Atlantic competition between the colliders is fast and furious. The competition shows that the LHC is not a leap into the void but rather a development and continuity of systematic, ongoing efforts. From this point of view, it becomes clear that a laboratory is best considered within a system of laboratories and not in isolation as an atomistic or self-contained unit, as a number of outstanding studies have shown (Collins 2004; Galison 1997; Knorr-Cetina 1999; Latour and Woolgar 1986; Traweek 1988). With the impetus of outward competition, the thrust of inner cooperation could not be far behind. The sense of helplessness gave way to that of urgency. The schedule and the strategy of repairing the damaged accelerator took the spotlight. The CERN web pages began publishing daily updates on the repair status.

Here a landscape starts emerging, which opens a window onto key anthropological concerns structured around the relation of temporality and the cycle of work. It compels us to ask if a breakdown might contain the possibility of discovering the logical determination of material activity in science. Marx showed that the objectivity of material production did not reside in its materiality, but in its form (Baudrillard 1981). The same recognition must be extended to the consideration of a laboratory. Any analysis of the interior world of the laboratory must not be based solely upon an inventory of objects, but on the distribution of work, the organization of labor, in relation to instrumentation. The next section outlines some of the key elements of expertise, manpower, equipment, technology and intellectual specifications of the instrument in reference, the LHC, together with the requirements for its initial operation, which acquaint us with the distribution of work in the laboratory and the conditions under which science is produced. 


\section{Machine parameters and accelerator physics}

The LHC Project was ratified by the CERN Council, the highest authority of the organization comprised of 20 European Member States, in 1994, with approval for the construction of the 14-TeV accelerator coming through in December 1996. The substantial resources required in building the colossal accelerator led to the involvement of nations outside of CERN member states including Canada, India, Japan, Russia and the United States. The Conceptual Design Report, or the "Yellow Book," specifying the chief parameters of the machine such as the superconducting magnets, RF and beam feedback, collimation and other systems, was brought out in 1995. While the basic design of the Yellow Book remains unaltered, substantial modifications in engineering and hardware were introduced over the years as accelerator science and technology kept advancing in energy regimes, precision, performance and complexity (Bruning et al. 2004; Evans 2009). ${ }^{6}$

When we consider the chronology of accelerator development, we observe an interesting feature that opens up a perspective on the relation between intellectual demands of science and the material conditions of instrumentation (Chao and Chou 2008; Livingston and Blewett 1962; Sessler and Wilson 2007). In the particle physics community, the LHC is often referred to as a "discovery" machine, designed to push the energy frontier and gain new insights into the sub-nuclear world. In contrast, the proposed next-generation collider, the Compact Linear Collider, or CLIC, is termed a "precision" machine, which would explore in depth and substantiate in detail what the LHC discovers. The collider at CERN prior to the LHC, the Large Electron-Positron Collider, or LEP, had been designed to measure the masses of $\mathrm{W}$ and $\mathrm{Z}$ particles to an extremely high accuracy. The $\mathrm{W}$ and $\mathrm{Z}$ particles themselves had been discovered in 1983 at an earlier CERN collider, the Super Proton Synchrotron or SPS.

In a nutshell, the development of accelerator systems shows a very interesting alternating logic of expansion and consolidation, or discovery and validation. Mysteries unraveled in one generation of accelerator are exploited for further probe and precision by the next-generation accelerator (Brunning and Collier 2007; Giudice 2010). The complementarity of this alternating logic reveals to us the grounds on which CERN developed the LHC, an instrument that is expected to open a window onto new discoveries of matter and energy, right after precision

\footnotetext{
${ }^{6}$ Articles appearing in the issues of the CERN Courier, CERN's monthly journal, provide a systematic account of different phases of the LHC design and development process, such as "French green light for LHC civil engineering" (October 1998), "Model magnet for CERN's LHC reaches 250 T/m in Japan" (April 1999), "First test beams are delivered for the LHC" (September 2000), "Going into the cold: LHC systems reach an important milestone" (December 2001), "High-energy accelerators look to R\&D" (June 2001), "Last LHC magnets from Siberia reach CERN" (September 2001), "CERN reacts to increased LHC costs" (January/February 2002) and so on. I have relied on issues appearing between 1994 and 2007 of the CERN Courier for much of the history of the LHC accelerator.
} 
Fig. 4 Radiofrequency cavities in the LHC cavern

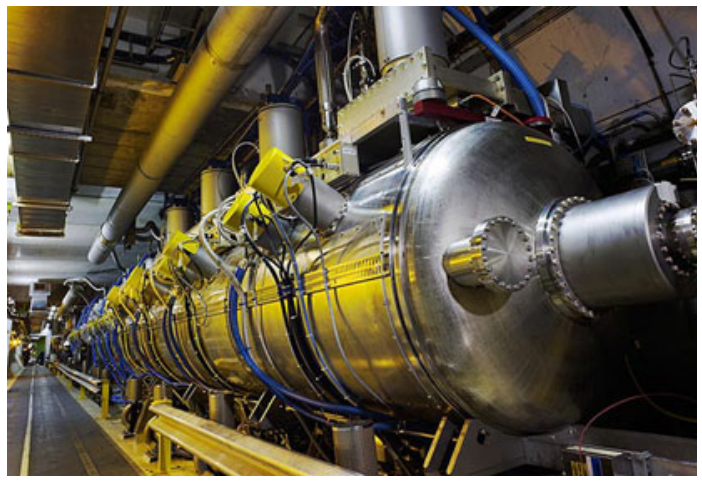

measurements were carried out by the previous accelerator, the Large ElectronPositron Collider. ${ }^{7}$

This pulsating logic of discovery and precision leads us to a conception of knowledge not so much as a vision of fixed forms, "as a production" (Althusser and Balibar 2009: 24) alive to activity and spontaneity. With this historical background of accelerator development, it becomes clear how the LHC, a global collaborative effort dedicated to discoveries, decisively moves in the realm of the possible and stretches the existing range of technologies to the limit. To begin with, its design performance envisages roughly 30 million proton-to-proton collisions per second, spaced by intervals of $25 \mathrm{~ns}$, with center-of-mass collision energies of $14 \mathrm{TeV}$, which is seven times larger than those of any previous accelerator. In order to achieve such rapid acceleration, the machine uses advanced "radiofrequency cavities" or dynamically changing electric fields at a radio frequency of 400 megahertz $(\mathrm{MHz})$, which push particles to ever-higher energies, much as ocean waves help a surfer gain speed, until the protons are whirling around the accelerator at some 11,000 times per second (Breskin and Voss 2009; Evans 2009; LHC Design Report 2004) (Fig. 4).

While electric fields are used for particle acceleration in the longitudinal plane, magnetic fields are needed for transverse bending, steering and focusing the particle beams into very precise orbits with the aim of optimizing collisions (Bruning et al. 2004; Evans 2009). Magnets with transverse fields operating in superfluid helium at $1.9 \mathrm{~K}\left(-271{ }^{\circ} \mathrm{C}\right)$ make up the backbone of the LHC. These are known as "dipole" magnets and are used for deflecting or bending the particle beams along the $27-\mathrm{km}$ circumference (Fig. 5). Although particle motion under the influence of dipole

\footnotetext{
7 The types of particles used for collisions, such as leptons or hadrons, also play a role in the alternating and complementary logic of accelerator development. Hadron colliders, using protons or neutrons, are especially suited for discoveries as they allow for breadth in understanding the inner constitution of matter. Lepton machines that use electrons, positrons, etc. are more appropriate for precision measurements and in-depth probe of particles after their discovery. For lack of space, I am unable to go into the details of particle instrumentation, the history of accelerators, or the link between specific laboratories and instruments in the field of high-energy physics. For a review of the general principles of accelerator technology and the growth of accelerators over time, see Chao and Chou 2008; Livingston 1969; Livingston and Blewett 1962; Persico et al. 1968. Laboratory-specific histories of instrumentation technology are also available involving Fermilab (Hoddeson et al. 2008), Lawrence Berkeley National Lab (Heilbron et al. 1981) and Oak Ridge National Lab (Johnson and Schaffer 1994), to name a few.
} 
Fig. 5 Dipole magnet on a test bench

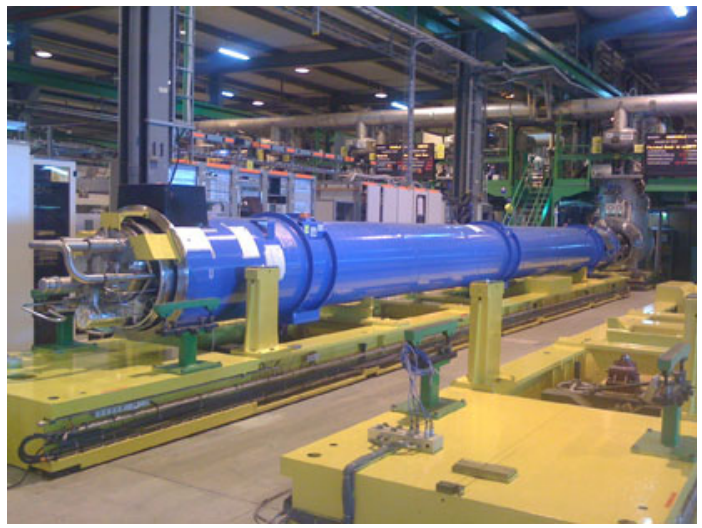

magnets is largely stable, it needs extra focusing to force the particles to remain on the "central" trajectory. Magnets used for squeezing or focusing particles closer together so as to increase the chances of collision are termed "quadrupoles." In all there are 858 quadrupole magnets and 1,232 dipole magnets located in the eight arcs of the LHC ring, with each of the dipoles having a length of $15 \mathrm{~m}$. Interestingly enough, "the 15-m physical limit to the dipole length was determined by the maximum length allowed by regular transport on European roads," while the maximum operational field was fixed at 8.3 Tesla "which has its roots in the realm of quantum mechanics rather than in European Union regulations" (Evans 2009: 74). ${ }^{8}$

Here we arrive at an interesting juncture of the LHC saga, one where we begin to observe how engineering considerations work alongside local operational conditions (Evans 2009; Breskin and Voss 2009). These conditions are negative in the sense that they designate less what the features are than what they are not. The chief problem facing the Hadron Collider during design and construction was the following: the maximum energy attainable in a circular accelerator depends on the product of the bending radius in the dipole magnets and the maximum field strength attainable. In order to bend two particle beams and generate field strengths in opposite directions, a large area is required. However, the diameter of the underground tunnel-where the collider was to be housed-at 3.8 meters posed a severe constraint. Constrained by the size of the actual tunnel, it was deemed impossible to fit two completely independent rings of the collider. The problem was daunting. How to confine two counter-rotating proton beams, that is, two separate magnet apertures, with opposite field orientations into the 3.8-m diameter of the LHC tunnel? ${ }^{9}$

\footnotetext{
8 The construction, transportation and installation of the dipole magnets, which had to cross a number of logistical, civil engineering, legal and financial hurdles, is a fascinating story in itself. See in particular the articles from CERN Courier, "LHC dipole production begins to take off" (January 2004), "LHC dipole installation gets to half-way mark" (September 2006), "The longest journey: the LHC dipoles arrive on time" (October 2006) and "The last dipole makes its descent" (June 2007), which give a glimpse into the magnet development process.

9 CERN had taken the decision that the LHC was to be constructed in the LEP tunnel for optimum use of existing infrastructure and keeping costs down, which posed a number of constraints on the LHC machine design and layout. See Evans 2009 for more discussion.
} 


\section{CROSS SECTION OF LHC DIPOLE}

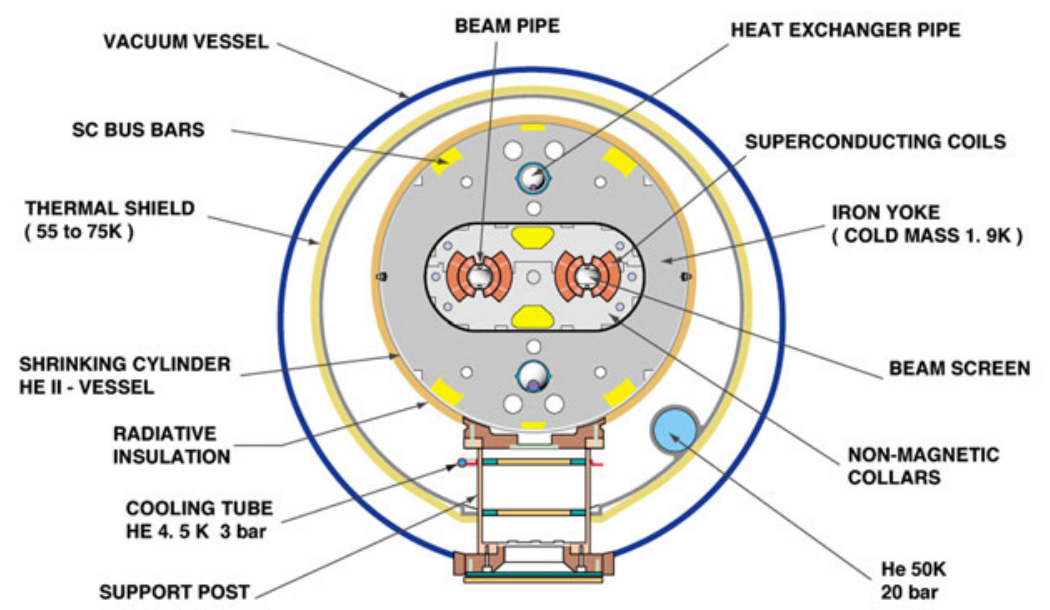

CERN AC HE HO7A V V2/02/98

Fig. 6 Cross section of the two-in-one LHC magnet structure

The grave challenge of space limitations in the tunnel, and the need to keep capital and operating costs down, led the team of accelerator physicists and engineers spearheaded by Lyn Evans, the celebrated "LHC Project Leader," to the adoption of a novel "two-in-one magnet" design for the LHC. The basic idea of a two-in-one magnet system is that windings for the two beam channels can be accommodated in a common cold mass cryostat since magnetic flux is circulating in opposite directions in the two channels (Fig. 6). At the same time, the design provides a compact structure that fits two separate beam apertures into the relatively small existing machine tunnel. However, this makes the magnet structure extraordinarily complicated and challenging because the separation of the two beams has to be small enough so that they can be coupled both magnetically and mechanically. In the LHC, the two beam pipes are separated by a mere $19 \mathrm{~cm}$ inside a common iron yoke (which returns the magnetic field) and the cryostat.

This inaugural move made by the LHC is certainly a radical one. As Lyn Evans explained to me, "this is the first time that the two-in-one magnet design has been built, so there is no existing experience to build on. The concept of a two-in-one magnet goes back to renowned accelerator physicist, Bob [Robert] Palmer, of Brookhaven. But nobody had used it.... In the late 70s we decided to use it. It made perfect sense for the LHC because it is a $\mathrm{p}-\mathrm{p}$ [proton-to-proton] collider and so the magnetic field is up in one aperture and down in the other aperture. You couldn't get this to work with $\mathrm{p}-\overline{\mathrm{p}}$ [a proton-anti-proton collider]. The requirement that the fields must be in opposite directions in the two apertures [for a proton-proton collider] ensures that there is no 
saturation of the central part of the yoke. This simple geometry of flux lines makes possible the exquisite design of a two-in-one dipole structure for the LHC." Indeed its uniqueness among accelerators has earned the LHC the befitting epithet, "Lord of the Rings," and Evans has been its principal person in charge for 14 years, involved right from conception and design through to construction and operation stages. The evolution of the machine had achieved its goal, with growing consciousness and success, when it began operation on September 10, 2008.

With this as our point of departure, we can now inquire into the incident of the sudden helium leak that struck the accelerator barely days after it started operation in order to see what it foregrounds and what it tends to screen out. Might not one discover in the unfortunate incident some constituent principle of science? Might not crises or breakdowns find a positive evaluation in disclosing the intellectual nerve of instrumentation that connect these with specific principles of knowledge and classification? ${ }^{10}$ By calling for a positive evaluation, I do not mean a question involving the perfection or purposiveness of instrumentation, but the need to isolate its originality in the multiplicity of activity, to interrogate it as a language, or a discourse, to find an Archimedean point that discloses something of its intellectual character and inner force. The objective may seem less mysterious if conceptualized as a dynamic process, built around the contrast of the normal and the exceptional, which reveals the configuration of the machine in the interface of temporality and normativity. This is its interest for ethnography. So if we pick up the thread of our story where we left off before the general intermission, namely, with the period immediately after the explosion when repair work on the accelerator took the spotlight, we are led to a curious moment of rupture, a break, which accentuates the difference and separation of the various units or sub-cultures of high-energy physics (Galison 1997; Knorr-Cetina 1999).

\section{Event and structure}

The period immediately following the September incident reveals an interesting moment in the life of a laboratory: while efficient and successful organization of work accentuates the nature of coordination existing between various specialized units and groups, a failure or a crisis manifests how the collectivity disperses and each technical component or unit settles or coheres into its own niche. In saying this, I am not trying to imply a sense of malfunction or a lack of discord between distinct units. What is simply meant is that during a breakdown, relations between various specialized units have decomposed, each functioning separately and autonomously, and fulfilling the task it is responsible for. Whereas cooperation is generally carried out in a spirit of self-consciousness and propaganda, the work done alone is really a legitimate fulfillment of the same impulse, only not glaring to be somehow perceived by all.

\footnotetext{
${ }^{10}$ Professor Jit Singh Uberoi brought before my attention the critical significance of this moment of crisis that had affected the laboratory. It is his insight on temporality, and its singular importance to fieldwork and anthropology, which I have developed in the present essay.
} 
In other words, cooperation or teamwork is sought as beneficial and propitious; it promotes harmony and leads to the completion of tasks in the acts of exchange. However, a crisis, when it occurs, triggers an awareness of the artificiality and the inadequacy of community and communication. The sense of exigency, the strain of repair, to seize hold of the details, all justified by an immediate purposiveness or a goal in sight, made it necessary for individual units of the laboratory to function on their own with little connection to the whole or to neighboring parts. This interest in the separation and autonomy of tasks is easily justified when we focus on the period between September 19, 2008, the day when the collider stopped, and October 23, 2009, when it resumed operation. During this period, each sub-culture of physics, namely theory, experiment and instrumentation, largely revolved on its own pivot, as I will show now.

Without exciting new collision data, there was little for theoretical physicists to do. Their discussions and seminars were carried out without liveliness. The conveners of the "Joint EP/PP" seminars began organizing talks on issues peripheral to physics such as "The Evolution of Religious Beliefs" (August 13, 2009) or "The Strange Friendship of Pauli and Jung-When Physics Met Psychology" (December 10, 2009). Their justification was, as Luis AlavarezGaume, a theoretical physicist and one of the conveners explained, "Once the LHC starts, the entertainment would stop." Until then, they claimed, they were aware of "killing time."

Most of the experimental physicists went back disappointedly to Monte Carlo (or simulation) data and cosmic data. The few days of operation before the incident had offered them a glimpse of the potentiality of the machine, so they seemed particularly affected, haunted and dejected by the intervening delay. Their main concerns were the steady accumulation of data by the rival Tevatron Collider, the delays in their own and their students' careers, the possibility of other mishaps occurring in a machine so big and novel and the dangers of losing support of funding agencies for the future experiments.

In complete contrast to the theoretical and experimental physicists were the accelerator physicists and engineers, energetically on their toes, removing, transporting and repairing the damaged magnets, manually checking over 150-magnet interconnections in the five warm sectors of the LHC, carrying out cleaning operations in the vacuum chamber in sector 3-4, installing new "DN200" relief valves in half of the machine, and reinforcing the support of 100 main quadrupoles to provide for their better anchoring to the ground. Key to the engineering work in this period was the installation of a new magnet "Quench Protection System" throughout the machine to prevent similar disasters in the future. All this repair and testing work was being carried out under the scrutinizing eyes of the whole organization.

During this time, there was no rhetoric, with sentimental overtones of progress and achievement, advocating the harnessing of work toward a common goal. Instead what confronts us is the total division and separation between the demands of physics and engineering. The separation between the demands of physics and engineering pertains to the performance and safety of the machine. The engineering point of view expressed doubts and fears regarding the actual operation of the 
instrument, whereas the physics point of view favored the potential of the instrument, the results to be derived, that is, high-energy collision data for which the instrument had been assembled. Most experimental physicists seemed in favor of immediate running of the LHC so that they would have some amount of collision data to work with and effectively stay in competition with the Tevatron. The accelerator physicists, on the other hand, had a more cautious approach. The vast majority of them preferred carrying out exhaustive repairs, before recommissioning the collider, to rule out the possibility of such incidents occurring in the future.

In February 2009, the annual "LHC Performance Workshop" was held in Chamonix, France, to critically review the schedule of repair and consolidation work on the LHC, which involved prominent experimental and accelerator physicists. The chief question debated at the workshop was: At what beam intensity should the Hadron Collider be run? Two key alternative scenarios were presented. Scenario one involved the installation of necessary spare parts, the "DN 200" pressure relief valves, in the 4 (warm) sectors and commencing collisions as quickly as possible. This, however, meant that the machine could only deliver limited performance, running in the range of $3.5 \mathrm{TeV}$ energy per beam, instead of the stipulated $7 \mathrm{TeV}$ per beam. The low energy run would be followed by a yearlong shutdown to conclude the remaining repairs and installations, and moving onto the specified energy level of $7 \mathrm{TeV}$ per beam. Scenario two involved installing the relief valves in all the 8 sectors of the LHC, undertaking diagnostic tests on the whole machine, going for a delayed start, but at full energy of 7 or $8 \mathrm{TeV}$ per beam. The motivations to the two scenarios cut across various issues such as: Was it possible to minimize the impact of the delay and stay in competition with the Tevatron? How could machine safety be weighed against the need for experimental data? Could the collider be protected against future incidents in a foolproof way?

The alternative scenarios were thoroughly discussed at the Chamonix workshop. On the whole, experimental physicists favored the first scenario, namely, a limited run with reduced energy in the immediate, followed by a longer shutdown. On the other hand, accelerator physicists pleaded for time and caution to undertake comprehensive repairs and checks. They tended to emphasize the "lessons learnt" from the September incident and came up with intricate lists of priority issues that needed to be addressed before recommissioning the LHC in any haste. ${ }^{11}$ Needless to say in a situation so complex, the lines of fission and fusion were not completely rigidly drawn. Speaking right after the workshop, the Director of Accelerators and Technology, Steve Myers, remarked that midway through the workshop he grew skeptical and switched from favoring the second scenario to the first and again back! Significantly, the workshop ended, as Meyers reported, with "no consensus in Chamonix."

The controversy is interesting because it shows that the organization of science is not a homogenous ensemble, but marked by a sharp division between theory, experiment and instrumentation, at the heart of which lies the institutionalized opposition of pure and applied, or science and engineering. It is this opposition and the consequent legitimacy of each respective domain's demands that makes it

\footnotetext{
11 From Steve Myers' presentation on 24th February 2009 summarizing the Chamonix Workshop.
} 
possible to identify the meaning and the use to which the instrument is put. The assessment of the possible research outcomes (meaning) for which the instrument has been assembled falls under the purview of physics. The conditions, which must be obtained before the instrument can be exploited for research potential (use), belong to the domain of engineering. Before elaborating this distinction or its interpretation as a general fact, two observations are in place. First, the distinction of pure and applied must be analyzed in structural as well as in functional terms (Habermas 1973; Heidegger 1977). In fact, the functional consequences that arise from the division between pure and applied often conceal the more structural aspects of the distinction. This will be examined in a later section.

The second observation pertains to the recognition of diachrony and the developmental cycle of instrumentation. Any assessment of the general organization of labor in high-energy physics is inadequate without an approach that incorporates the tempo of laboratory work, especially its transition moments, which have something interesting to reveal. Transition moments help us understand the critical link between general principles and immediate problems (Marx 1964; Althusser and Balibar 2009). This inquiry into diachrony and transition, not simply as an ingredient of narrative or a description of reality, but as a component of conception and analysis, I will pursue next.

\section{Transition and the new normal}

The section attempts to probe the following question: How should we interpret the incident in September and the resulting suspension of the machine? In effect we are asking what is the specificity of this event? A question about specificity implies difference, such as what makes this period different from other periods? I will use the concept of periodization developed by Marxist scholars, Althusser and Balibar (2009), which presents us with a framework alternative to the continuity of evolution, with the transition of structures, their inner tendencies and contradictions, and their relative autonomy. The period just after the September 19 incident cannot be interpreted as a mere unfolding of a succession of events, but forms a great inward drama to which one can unequivocally apply the term "transition" or "liminal." It expresses an orientation-precisely that of a transition from one mode to another. A serious analysis must take into account the distinct phases and the tempo of work, in and through which units and actions cohere or go apart, with the temporal direction being critical in which the structuring of events takes place: events such as before, after or transition.

The importance of temporality and periodization in the sense of Marx's "development of form" (1964) can also be substantiated from the concept of liminality, made familiar to us from the study of religion and ritual. A great deal of anthropological writing has been concerned with elaborating the scheme outlined by van Gennep (1960) of the phases of ritual action in terms of separation, liminality and reaggregation. A "betwixt and between" moment, liminality represents a midpoint, a transition in a sequence of events. It forms a socially and structurally ambiguous stage, which heralds the restoration of social order. People in liminal 
moments often feel disconnect with the preceding and succeeding moments. Although the attributes of the liminal phase are necessarily ambiguous, since they elude through normal classifications, it is a phase that is "institutionalized and preordained" (Turner 1969).

In availing the concept of liminality, I am trying to suggest that in discussing scientific work life, it is essential to be aware of the distinct stages that mark the developmental cycle of instrumentation, especially the transitional stages. For the characteristic feature of the transitional stage is that it possesses the source and substance of a system. According to Turner, "liminality may perhaps be regarded as the Nay to all positive structural assertions, but as in some sense the source of them all, and, more than that, as a realm of pure possibility whence novel configurations of ideas and relations may arise" (1967: 97). Thus understood, Turner's account of the liminal as disclosing the kernel of a system suggests an extraordinarily ambitious program of meaning and analysis. However, as opposed to Turner, I am arguing that the liminal need not be a manifestation of "blurred boundaries," one where structural bonds have dissolved and homogeneity prevails. On the contraryand this is important - the transitional period can express with remarkable clarity the boundaries and separations between events, actions and persons. That I am still using the term liminality as an aid to grasp the scheme of science is not forsaking the term of its chief merit. The liminal, in the sense of emphasizing the theme of inversion, clearly marks the period just after the incident in September 2008.

Prior to the incident, the distinct sub-cultures of physics exemplified concerted action; feedback from each distinct unit of the laboratory was seen as aiding and enabling the work of another. This element of conjunction and coordination is well recognized (Traweek 1988). However, during the suspension of the accelerator, the boundaries and the separation between the units became visibly accentuated: theory, experiment and instrumentation dispersed into their respective niches. During this time interval, many new features to the machine were discovered, including hitherto unforeseen flaws, which could critically disrupt the future operation of the accelerator. Stock was taken of some of these, and others were set aside for later when the LHC would require substantial upgrades for running at optimum energy of $7 \mathrm{TeV}$ per beam (CERN 2008). After the incident, repairs and subsequent consolidation work took nearly a year to complete.

The year 2009 was the year of transition in the life of LHC when unstinting engineering efforts on repairs, consolidation, hardware commissioning and preparations for the beams finally came to an end and physics took the thrust. On October 23, 2009, particles entered the LHC for the first time since the September incident, and soon after the collisions began. On November 30, 2009, the LHC became the world's highest energy particle accelerator when protons in each beam reached an energy of $1.18 \mathrm{TeV}$. This exceeded the previous world record of 0.98 $\mathrm{TeV}$, which had been held since 2001 by the Tevatron Collider at the Fermi National Accelerator Laboratory, USA. By the end of the year 2009, the transition had successfully been made from repair and commissioning of the accelerator to its exploitation for physics. A switch had taken place. For the community as a whole, now physics took the spotlight, with the accelerator receding to the background. 
In rich and substantiated ways, the emerging field of study called STS, or Science and Technology Studies, has drawn our attention to the significance of periodization and temporality in mapping the material culture of a laboratory. A particularly interesting analysis of periodization in instrumentation is found in Pickering's works $(1984,1995)$. He insists on the recognition of temporal emergence and historicity of knowledge in evaluating the pursuits of science. In particular, he raises the question: "How should we conceptualize temporally emergent phenomena?" (1993: 561). Peter Galison provides an excellent narrative of critical episodes in physics where the decision to end an experiment is motivated by a complex of interlaced factors. He uses the term "intercalated periodization" (1999) to describe the various factors, like theoretical presuppositions, elimination of background or calculation of systematic errors, all of which critically influence the decision to accept a result and end an experiment. Allan Franklin's account of the discovery of parity non-conservation highlights the uneven temporality of experimental outcomes and theoretical activity (1986). The idea that science develops in neat, regular or predictable ways receives a drubbing in the works of most serious historians of science.

While these approaches have given careful consideration to issues of temporality in high-energy physics, especially to moments of emergence and end (Galison 1987; Pickering 1984), they have shed little light on the liminal stage as an autonomous periodization worthy of study. As I have attempted to suggest, in discussing scientific work life we should pause and reflect on an incident's specificity, which accommodates its liminal status. There is a great difference between simply following an event in its temporal course and being able to distinguish the different phases of temporality and understanding them conceptually (Althusser and Balibar 2009). In analyzing the organization of science, we are not dealing with the study of merely empirical questions, for indeed the empirical validity of different phases is well recognized by informants themselves, but rather with the epistemological problem of what conditions underlie the operation of work in science?

As long as we are given to the contemplation of temporality as an empirical generalization, as a flow of events, the direction of movement in science cannot be discerned, and analysis remains at the level of description. When we focus on time, not as duration, but as a paradigmatic ordering of events, that is to say its content is grounded in specific cultural or normative constructs, we observe the production or mutation of knowledge not solely as products or as results of a movement, but as "the appropriation of the real world by different practices, theoretical, asthetic, religious, ethical, technical, etc." (Althusser and Balibar 2009: 71). In other words, time is not an isolated or even an intrinsic feature, but part of a broader cultural determination and subject to structural social relations (Bourdieu 1977; Turner 1969).

The key conclusion that follows from this conception of time and periodization is that it not only allows us to escape the linearity of time, but also helps to destroy time "as a framework or common support for every possible historical determination" (Althusser and Balibar 2009: 329, italics in original). Once we refuse the obviousness of time, or to presuppose a reference to time as an a priori, the space opens up for transition periods to appear in their specific forms, and the variations of 
these forms, as constituted by abstraction and structural relations (2009: 337). Such a model of temporality and periodization allows us to grasp the uniqueness of distinct temporal frames or phases by engaging with material and social conditions internal to the object of study, such as distribution of work, mobilization of labor, etc., without eliminating general concepts and principles of knowledge and classification.

Focusing on the accelerator in the interim period, that is, just after the September 2008 incident and before being recommissioned for operation in the year 2010, we find the total division and separation between the domains of science and engineering, as I indicated above. The question now arises where does the period of transition stand vis-à-vis the complex structure of high-energy physics and what does the division of science and engineering express about its inner constitution. To address this question, we must take recourse to the division of labor that governs the organization of tasks and capacities in contemporary particle physics, which I have alluded to in the discussion but not elaborated upon so far. The exposition of the division of labor is the subject of our attention in the following pages. With this we enter the final stage of analysis.

\section{The division of labor}

To comment on the division of labor is difficult now when everything has already been examined on this topic and a saturation point is nearly reached (Gouldner 1954; Rueschemeyer 1986; Smelser 1963; Sullivan 1995). Instead of dwelling on its more general aspects, I wish to consider a very specific question: In what way does the division of labor follow from the demarcation and separation of the spheres of mental labor and manual labor, or theory and practice? As Marx et al. suggest, the "division of labor only becomes truly such from the moment when a division of material and mental labor appears" (1947: 20). In this respect, it is worth examining how the opposition of mental labor and material labor, or theory and practice, expresses itself in the institutionalized division of labor and is bound up with the technical operations of a laboratory.

At present CERN is sub-divided into eight departments. These are listed below with the names of the department heads alongside:

PH Physics: Philippe Bloch

IT Information Technology: Frederic Hemmer

BE Beams: Paul Collier

TE Technology: Frédérick Bordry

EN Engineering: Roberto Saban

HR Human Resources: Anne-Sylvie Catherin

FP Finance and Procurement: Thierry Lagrange

GS General Infrastructure Services: Thomas Pettersson

The preeminent department is Physics. It carries out basic scientific research in theoretical and experimental physics. The departments of Beams, Technology and Engineering are responsible for the hardware of the accelerator, such as the 
production and testing of magnets, cryogenics, beam instrumentation, etc. The other departments, like Human Resources or Finance and Procurement, provide infrastructure support and services. Myriad gradations of personnel are found in any stream or branch. People who design "detectors" are distinguished from those involved with computing or data analysis; those working on the magnets of the accelerator are far removed from the elaborations of string theory, and so on. While immediate observations may lead us to emphasize the heterogeneity of tasks, now it remains to say something about their internal coherence. To be sure, broad generalizations about the distribution of work may appear dangerous because exceptions can be found to any aspect of classification. However, when we fall upon the evidence of sustained fieldwork, a few distinctive principles are discernible. One of these principles, and its intellectual force and meaning, I will try to identify from my own fieldwork data to show how it forms a possibility condition of laboratory science, and which allows for the interpretation of events in their singularity, in their movement and in their effects.

During fieldwork, I observed most informants attesting to an implicit hierarchy at the root of the intricate diversity of tasks and personnel with theoretical physics more or less at the top of this informal hierarchy, with experimental physics a close second, followed by accelerator physics and engineering, and the technical and the administrative workforce widely perceived as auxiliary staff. Although I received indirect answers from informants as to why theoretical physics should rank higher than accelerator physics or experimental physics lower than theoretical physics, I was gradually able to piece together their main ideas on the apparent hierarchy to look beneath to the power which fashions it, the operative demarcation of pure and applied, or between those who "think" and "those who use their hands," as one of my engineering informants, Francesco Bertinelli, put it. While the anti-intellectualism of engineering is somewhat exaggerated by Bertinelli, who is himself an astute intellectual, the economy and repetition with which I encountered the institutionalized distinction of pure and applied in the everyday organization of work, in the division of labor, is an indication that it has an intelligible pattern and methodological validity.

Since the full role and scope of this distinction lies in everyday work, without whose description it is not made clear, let me consider that at once. For instance, in the field of theoretical physics, those who develop models, theoretical frameworks or mathematical techniques, are defined by most informants as engaged in "model building" or "pure theory," and are distinguished from those who specialize in "computations," the implementation of algorithms necessary for model building work or "phenomenology," where predicted values from theory are compared with experimental data. Phenomenology or computations are distinctly perceived by most informants to be in the zone of "application."

In the experimental physics community, which is numerically preponderant than the theory group, a distinction is commonly observed between those involved with installation, commissioning and operation of hardware or the detectors, designated as "applied physicists," and those involved with data preparation and analysis termed "research physicists." Processing and analyzing physics data is deemed more interesting than constructing detectors or installing cables. The attribution of 
differential values to mental and manual labor is often conveyed in the anxiety of a number of informants working on the hardware of experiments if they could make a transition to the more exciting task of data analysis in the course of their careers. In 2009, after 19 years of working on the "end-cap B" of the CMS detector, David Cockerill, of Rutherford Appleton Laboratory, was both happy and anxious that the detector was completed and sealed. He was happy because he had contributed in no small measure toward its completion. Anxious because what does he do now? When I met him a year later, he cheerfully told me, "I have moved to data analysis...not easy, I have done it."

In the accelerator sector, likewise it was impressed upon me that "TE" (Technology Department) was the leading arm since it involves research and conceptualization of the star project, the LHC and the future projects, while the "EN" (Engineering) department provides technical coordination and infrastructure support to the accelerator and the various experiments. Coordination, training and safety were considered "lower-end jobs, or what one does close to retirement," as a magnets engineer, whose name I shall not disclose, told me with some bitterness. "When one is young, one likes to think about the mysteries of the universe, play with ideas," he remarked. Conceptualization and development of technology is perceived as being more challenging or rigorous than implementation of safety procedures or finding "applications" of a given technology.

Having said this much, I hasten to add that the feature of hierarchy itself is the least of my concerns. I have a rudimentary idea of the hierarchy without anything substantial to offer by way of analysis. My particular interest lies in surveying the division of labor from the standpoint of the classification of knowledge where the opposition of theory and practice, or pure and applied, comes to form the heart of the division of labor, as it was disclosed to me during fieldwork. Far from being accidental to the activity of high-energy physics, the opposition of theory and practice functions in the institutionalized division of labor as its indispensable constitutive principle and defines principal aspects in which creativity is exercised and work is discharged. In other words, talents and tasks are a source, which in the course of time, must evolve into a distinction of manual activity or mental activity, that is, those who work with their hands, and those who use their minds, or multifariously into modeling, theorizing, designing or manufacturing.

My interest in the value of this distinction lies not in its achievements or consequences for the organization, but in what it signifies as an instrument and symbol of knowledge. As Habermas succinctly argues, "The technical and practical interests of knowledge are not regulators of cognition which have to be eliminated for the sake of the objectivity of knowledge; instead they themselves determine the aspect under which reality is objectified, and can thus be made accessible to experience to begin with" (1973: 9). Since informants in the community perceive the opposition of theory and practice as important, I have taken it up at some length. Above all, the instrument is an embodiment of the values of this opposition, and to which it is dedicated. The fact that I have isolated instrumentation as the site to expose the critical divides of mental and material, expressive and instrumental or real and possible, does not mean that it is the only one available for reflecting on the complexity of science. I have considered it because material culture is a key concern 
of anthropology (Keane 2003; Tilley 2006) and provides fertile grounds for comprehending analytical divides with practical activity.

Ian Hacking (1983) has famously commented on the "disunity of science" owing to the proliferation of specializations that we find in it, where every material phenomenon or artifact is produced by different tools and techniques. Stable laboratory science arises, he maintains, when theories, materials and laboratory equipment evolve in such a way that they match each other and are mutually selfvindicating. Such "symbiosis" is a held to be a contingent fact about people, our scientific organizations and nature (Cartwright 1983; Duprè 1993; Galison and Stump 1996; Pickering 1984). The medley of local and transitory coordinations encountered in science has generated arguments that tend to emphasize its fluid and elastic character. Pickering (1995) finds the structure of scientific practice to be a "mangle," which extends from instruments, substances, materials, to actors and practices. Galison (1997) invokes the language analogy — of pidgins and creoles and their "trading zone" - in the recognition of how "two groups can agree on rules of exchange even if they ascribe utterly different significance to the objects being exchanged; they may even disagree on the meaning of the exchange process itself. Nonetheless, the trading partners can hammer out a local coordination, despite vast global differences" (783). In this mode of reasoning, contingency prevails, but not in any drastic way for it is counterbalanced by functional coordination.

However, outside of historical contingency or functional coordination, if we still wished to know what relation do everyday practices or outstanding events bear to general principles of knowledge and classification, there is a possibility of an answer, one that requires attending to the relations of symbol and instrument, abstraction and perspective, concept and object (Marx and Engels 1967; Althusser and Balibar 2009; Uberoi 2002). For indeed an initial impulse may lead to the ready conclusion that science is wholly heterogeneous and without a center. But a sustained reflection discloses-and as the discussion revolving around the September 19th incident is meant to emphasize-that the opposition of pure and applied, or theory and practice, forms the source and the sanction of the institutionalized division of labor prevailing in high-energy physics. Without this recognition, the concrete ethnographic fact involving the September incident, namely that those branches of physics that deal with theory or analysis had little role to play when the collider was suspended while the engineering work moved roundthe-clock, would not make sense. The claim of "disunity of science" (Duprè 1993; Hacking 1983; Galison and Stump 1996) passes over in silence the mechanisms and the sources of this disunity, and is forced to fall back on descriptions such as hybridity, symbiosis, contingency, etc. in characterizing scientific activity (Galison 1997; Pickering 1984), which explain nothing, as Marx would urge, of the internal determination or the specific form that science takes.

From weekly colloquia to staff picnics, as I observed over two and a half years at CERN, the division of tasks is axiomatically maintained, especially the division of pure and applied or theory and practice. Far from reproaching or defending the way in which instrumentation is organized in the laboratory, I have merely wanted to examine what it tells us about the principles of high-energy physics. The unforeseen incident owing to the electrical fault and the resulting suspension of the Hadron 
Collider invite us to examine a fundamental problem in the classification of knowledge: How does a singular event, such as a crisis, express the distribution of knowledge in the laboratory? Unforeseen or singular events do not escape the regularity of established conditions but combine with them to exhibit the unqualified normativity of social thought and conduct (Douglas 1999; Gluckman 1958; Turner 1969). During routine operation, the distinct sub-cultures of physics work selfconsciously in tandem, alongside each other. However, during a crisis or a breakdown, the organization devolves into identifiable niches, with each functioning largely on its own pivot. It is not astonishing that the nature of distinctions should appear more strikingly during "betwixt and between" or transition phases, such as the period immediately following the incident in September 2008, and before renewed operation in 2010. Therefore, any analysis of the mutual relations of the different sub-cultures of physics must consider the different phases of temporality conceptually in order to extract the source, and mechanisms, by which the division of labor is organized in the laboratory.

\section{Conclusions}

The progressive transformation of nature according to the prescriptions of physis (knowledge), put into practice by techne (craft), which modern science demands, has led to the rich material culture and complex division of labor found in highenergy physics today. The conception, plan, design and construction of the LHC, and the four main detectors, took nearly two decades. The incident in September 2008, occurring just nine days after a successful start, plunged the physics community in a pall of gloom. Most of the accelerator physicists and engineers I spoke to during this period stated that the incident had given them a jolt. The incident had introduced to them the fragility and complexity of a Hadron Collider. "We always know that there is a lingering possibility that some technical problem, maybe even very small, can threaten operation anytime. The September incident was a critical and a painful lesson but not without useful consequences," thought Gijsbert de Rijk, from the MSC group (Magnets, Superconductors and Cryostats), CERN, and my key informant in the area of accelerator physics.

It would be a mistake, however, to read into this crisis or a breakdown merely a negative sense of malfunction or even a positive sense of what it accomplished for the organization. The viewpoint of functional coordination is much too narrow. Nor does the argument of structural disunity (of science) advance beyond the category of contingency. A scientific laboratory is neither simple nor enduring. It is a work space which provisions for the conduct of experiments, coordinates the testing, manufacturing and assembling of instrumentation, facilitates collaboration on the use of materials, models and techniques and develops initiatives on the R\&D of future projects. Without fetishizing it, we may say that the instrument forms a symbol, which orchestrates the conjunction and disjunction of the three main subcultures of high-energy physics. Both these aspects of conjunction and separationof tasks and personnel-belong to the same logic, only manifesting at different phases in the cycle of work, as I have tried to show. The separation of tasks came to 
a vivid expression when the colossal collider suffered a breakdown. On the other hand, the conjunction of tasks finds expression in the routine and successful order of everyday work.

What is interesting from the point of view of anthropological fieldwork is that while most informants bemoaned the suspension of work following the incident, the ethnographer had gained a remarkable opportunity, to observe nothing less than the source of rhythms and routines of techno-scientific work life. The September incident at the LHC directs our attention to the issue of diachrony or temporality, not as a phenomenon of irreversible succession or a juxtaposition of events, but rather to the recognition that all temporality has its roots in principles of social classification (Althusser and Balibar 2009; Uberoi 2002). In framing my inquiry into the material culture of science, I have adhered to this fundamental insight on the social character of classification and conduct. My task, the way I conceived it, was to traverse the inner relationship between principles of classification and everyday problems in order to clarify the form of knowledge that science stakes. By isolating the September incident that disrupted CERN's LHC, the essay arrives at a key moment in the life of the laboratory when the division and separation of theory and practice stand critically exposed.

Acknowledgments I am deeply grateful to Gil Gilchriese and Ian Hinchliffe from ATLAS-LBNL, and Francesco Bertinelli, Lyn Evans and Gijs de Rijk from the accelerator division at CERN for generously sharing their understanding of instrumentation with me. I am obliged to the Wenner-Gren Foundation's Dissertation Fieldwork Grant (\# 7758), which enabled me to undertake fieldwork at CERN. Continuous support has been provided by the University of California, Berkeley, in particular Professors Lawrence Cohen, Terry Deacon, Tom Laqueur and especially Paul Rabinow. I wish to thank the students at Berkeley for their enthusiasm in my field research which has enabled me to understand it much better. Finally, Professor Jit Singh Uberoi has lovingly and painstakingly given direction to the research project. To him I am eternally beholden.

Open Access This article is distributed under the terms of the Creative Commons Attribution License which permits any use, distribution, and reproduction in any medium, provided the original author(s) and the source are credited.

\section{References}

Althusser, Louis, and Etienne Balibar. 2009. Reading “capital”. London, New York: Verso.

Baudrillard, Jean. 1981. For a critique of the political economy of the sign. St. Louis: Telos Press, MO. Bourdieu, Pierre. 1977. Outline of a theory of practice. Cambridge [England]; New York: Cambridge University Press.

Breskin, Amos, and Rüdiger Voss. 2009. The Cern Large Hadron Collider: Accelerator and experiments. Geneva: CERN.

Bruning, Oliver, and Paul Collier. 2007. Building a Behemoth. Nature 448(7151): 285-289.

Bruning, Oliver, et al. (eds.). 2004. LHC design report. Geneva: CERN.

Cartwright, Nancy. 1983. How the laws of physics lie. Oxford; New York: Clarendon Press; Oxford University Press.

CERN Annual Report. 2008.

Chao, Alexander W., and Weiren Chou (eds.). 2008. Reviews of accelerator science and technology. Singapore; Hackensack, NJ: World Scientific. 
Collins, Harry. 2004. Gravity's shadow: The search for gravitational waves. Chicago: University of Chicago Press.

Douglas, Mary. 1999. Implicit meanings: Selected essays in anthropology. London, New York: Routledge.

Duprè, John. 1993. The disorder of things: Metaphysical foundations of the disunity of science. Cambridge, MA: Harvard University Press.

Evans, Lyndon. 2009. The large hadron collider: A marvel of technology. Lausanne, Switzerland: EPFL Press.

Franklin, Allan. 1986. The neglect of experiment. Cambridge [Cambridgeshire]; New York: Cambridge University Press.

Galison, Peter. 1987. How experiments end. Chicago: University of Chicago Press.

Galison, Peter. 1997. Image and logic: A material culture of microphysics. Chicago: University of Chicago Press.

Galison, Peter. 1999. Reflections on image and logic: A material culture of microphysics. Perspectives on Science 7(2): 255-284.

Galison, Peter, and David J. Stump. 1996. The disunity of science: Boundaries, contexts, and power. Stanford, CA: Stanford University Press.

Gell, Alfred. 1992. The anthropology of time. Oxford: Berg.

van Gennep, Arnold. 1960. The rites of passage. Chicago: University of Chicago Press.

Giudice, Gian. 2010. A zeptospace odyssey. Oxford, New York: Oxford University Press.

Gouldner, Alvin. 1954. Patterns of industrial bureaucracy. Glencoe, IL: Free Press.

Gluckman, Max. 1958. Analysis of a social situation in modern Zululand (Manchester [England]: Published on behalf of the Rhodes-Livingstone Institute by the Manchester University Press.

Habermas, Jurgen. 1973. Theory and practice. Boston: Beacon Press.

Hacking, Ian. 1983. Representing and intervening: Introductory topics in the philosophy of natural science. Cambridge, New York: Cambridge University Press.

Heidegger, Martin. 1977. The question concerning technology, and other essays. New York: Harper \& Row.

Heilbron, J.L., Robert W. Seidel, and Bruce R. Wheaton (eds.). 1981. Lawrence and his laboratory: Nuclear science at Berkeley. Berkeley: University of California.

Hoddeson, Lillian, Adrienne W. Kolb, and Catherine Westfall (eds.). 2008. Fermilab: Physics, the frontier, and megascience. Chicago: University of Chicago Press.

Johnson, Leland, and Daniel Schaffer (eds.). 1994. Oak Ridge National Laboratory: the first fifty years. Knoxville: University of Tennessee Press.

Keane, Webb. 2003. Semiotics and the social analysis of material things. Language and Communication 23(2\&3): 409-425.

Knorr-Cetina, K. 1999. Epistemic cultures: How the sciences make knowledge. Cambridge, MA: Harvard University Press.

Latour, Bruno, and Steve Woolgar. 1986. Laboratory life: The construction of scientific facts. Princton, NJ: Princeton University Press.

Livingston, M. Stanley. 1969. Particle accelerators: A brief history. Cambridge, MA: Harvard University Press.

Livingston, M. Stanley, and John P. Blewett. 1962. Particle accelerators. New York: McGraw-Hill.

Masco, Jospeh. 2002. Lie detectors: On secrets and hypersecurity in Los Alamos. Public Culture 14(3): 441-467.

Marx, Karl. 1964. Pre-capitalist economic formations. Translated by Jack Cohen. Edited and with an introd. by E. J. Hobsbawm. London: Lawrence \& Wishart.

Marx, Karl, and Friedrich Engels. 1967. Capital. A critique of political economy. New York: International Publishers.

Marx, Karl, Friedrich Engels, Roy Pascal, W. Lough, and C. P. Magill. 1947. The German ideology. International Publishers.

Munn, Nancy D. 1992. The cultural anthropology of time: A Critical Essay. Annual Review of Anthropology 21: 93-123.

Persico, Enrico Ezio Ferrari and Sergio E. Segre. Ed. 1968. Principles of particle accelerators. New York, WA: Benjamin.

Pickering, Andrew. 1984. Constructing quarks: A sociological history of particle physics. Chicago: University of Chicago Press. 
Pickering, Andrew. 1995. The mangle of practice: Time, agency, and science. Chicago: University of Chicago Press.

Rueschemeyer, Dietrich. 1986. Power and the division of labour. Stanford, Calif.: Stanford University Press.

Sessler, Andrew, and Edmund Wilson. 2007. Engines of discovery. New Jersey: World Scientific.

Smelser, Neil J. 1963. The sociology of economic life. Englewood Cliffs, NJ: Prentice-Hall.

Sullivan, William M. 1995. Work and integrity: The crisis and promise of professionalism in America. New York: Harper Business.

Tilley, Christopher (ed.). 2006. Handbook of material culture. London: SAGE.

Turner, Victor. 1967. The forest of symbols: Aspects of Ndembu ritual. Ithaca, NY: Cornell University Press.

Turner, Victor. 1969. The ritual process: Structure and anti-structure. Chicago: Aldine Pub. Co.

Turner, Victor. 1980. Social dramas and stories about them. Critical Inquiry 7(1): 141-168.

Traweek, Sharon. 1988. Beamtimes and lifetimes: The world of high energy physicists. Cambridge, MA: Harvard University Press.

Uberoi, J. P. S. 2002. The European modernity. New Delhi: Oxford University Press. 